\title{
NOX-mediated impairment of PDGF-induced DNA synthesis in peripheral blood lymphocytes of children with idiopathic nephrotic syndrome
}

\author{
Amal Al-Eisa ${ }^{1}$ and Gursev S. Dhaunsi ${ }^{1}$
}

BACKGROUND: Cellular oxidative stress, inflammatory responses, and immunogenic events are involved in pathogenesis of idiopathic nephrotic syndrome (INS); however, the exact mechanism remains unknown. We examined NADPH oxidase (NOX) activity and platelet-derived growth factor (PDGF)-induced DNA synthesis in peripheral blood lymphocytes (PBL) of patients with INS.

METHODS: PBL from 15 patients with INS and 15 age- and gender-matched controls were isolated, and enzyme activities of NOX, catalase, and superoxide dismutase (SOD) were measured along with the assay of malondialdehyde levels and bromo-deoxyuridine incorporation. Protein expression of NOX-1 was measured using western blot analysis.

RESULTS: Patients with INS had significantly $(P<0.01)$ higher NOX activity and increased protein expression of NOX-1 in PBL as compared with controls. Catalase and SOD activities were markedly lower with lipid peroxide levels significantly $(P<0.01)$ increased in patients with INS. Ex vivo DNA synthesis in PDGF-stimulated PBL was significantly $(P<0.01)$ reduced in patients with INS; however, diphenyliodonium, an inhibitor of NOX, markedly corrected impairment in growth factorinduced BrdU incorporation.

CONCLUSIONS: These results show that NOX activation might have a role in regulation of lymphocytic activity in patients with INS through the impairment of PDGF mitogenic function and might contribute toward pathogenesis of nephrotic syndrome.

I diopathic nephrotic syndrome (INS) is a common glomerular disease affecting both adults and children. Patients with INS respond favorably to steroids; however, a number of patients with INS may suffer from frequent relapses or develop dependence on or resistance to steroids in the later stage, leading to a protracted course $(1,2)$. Although exact pathogenic mechanisms of INS remain unclear, altered immunological responses, cellular oxidative stress, and genetic aberration have been proposed (3). Inflammatory cascades involving production of free radicals and their role in alteration of glomerular hemodynamics have also been suggested to have a role in pathogenesis of nephrotic syndrome (NS). Overproduction of reactive oxygen species (ROS) causes cell injury by lipid peroxidation, particularly affecting the structural integrity of the tubular epithelial cells $(4,5)$. Increased oxidative stress is believed to have harmful effects on renal tissue of patients with INS, and circulating cells, particularly those involved in immunogenic responses, may well be at risk of oxidative damage. It has been suggested in earlier reports that a decreased immunity and increased oxidative stress put children with NS at the risk of frequent relapse episodes (6). Lymphocytes and their function are known to be affected in children with INS, and a significant number of peripheral blood lymphocytes (PBL) have been reported to undergo apoptotic changes in patients with INS $(7,8)$. Oxidative stress has been previously demonstrated in various kidney disorders such as glomerulonephritis and acute renal injury including an important role for ROS in the pathogenesis of experimentally induced nephrosis $(9,10)$. Catalase, superoxide dismutase (SOD), and glutathione peroxidase (GPx) are the key antioxidant enzymes that degrade ROS hydrogen peroxide, superoxide anions, and lipid peroxides, respectively, and activities of antioxidant enzymes have been shown to get altered during the development of oxidative stress in children with INS (11). Besides several other sources of free radical production during cellular metabolism, NADPH oxidase (NOX), a complex multicomponent enzyme system, is responsible for the production of highly reactive superoxide anions. NOX, originally reported to be localized in phagocytes to generate superoxide anion as a microbicidal tool, is now known to be present in several cell types and tissues with tissue-specific NOX homologs for heart, liver, brain, kidney, blood lymphocytes, and blood vessels (12). NOX-mediated superoxide production has been reported to be vital for some physiological functions such as smooth muscle cell tone, vascular motility, and cellular defense against invading infectious agents (13), and activation of NOX has been reported widely to have a role in pathogenesis of carcinogenesis, atherosclerosis, chronic renal 


\section{Articles $\mid$ Al-Eisa and Dhaunsi}

diseases, hypertension, and diabetes through superoxide anion-mediated oxidative stress (14). NADPH oxidase is a key player in the regulation of overall cellular redox; however, it's possible role in pathogenesis of NS has remained unexplored particularly in relation to immunogenic cells. This study was conducted to examine any association of NOX-mediated oxidative stress with growth-factor activity in PBL of patients with INS that could provide new insights into lymphocyte-mediated pathogenic mechanisms.

\section{METHODS}

\section{Materials}

Cell culture medium, RPMI 1640, DMEM, and DMEM-Ham's F-12 were purchased from GIBCO (Grand Island, NY). Trypsin-EDTA, fetal bovine serum, and penicillin/streptomycin were procured from Sigma Chemical Company (St. Louis, MO). Bovine serum albumin (BSA), diphenyliodonium (DPI), and platelet-derived growth factor (PDGF) were purchased from Calbiochem (La Jolla, CA). All other chemicals and reagents were obtained from Sigma. Cell culture plates and petri dishes were obtained from Falcon Becton Dickinson (Oxnard, CA). Antibodies against NOX-1 and actin were procured from Abcam (Cambridge, MA). BrdU assay kit was purchased from Calbiochem.

\section{Patients}

Fifteen children with confirmed diagnosis of INS (Table 1) were recruited for this study. All were evaluated and managed at the pediatric nephrology unit in Mubarak University Teaching Hospital during the period from January to September 2015. Diagnosis of INS was based on the KDIGO guidelines with the presence of generalized edema, nephrotic-range proteinuria (urinary protein: creatinine ratio (u PCR) $\geq 200 \mathrm{mg} / \mathrm{mmol}$ or $3+$ protein on urine dipstick), and hypoalbuminemia $(\leq 2.5 \mathrm{~g} / \mathrm{l})(15,16)$. Relapse of nephrotic syndrome was defined as the uPCR $\geq 200 \mathrm{mg} / \mathrm{mmol}$ or $3+$ protein on urine dipstick for three consecutive days. Remission was defined as uPCR $<20 \mathrm{mg} / \mathrm{mmol}$ or $<1+$ of protein on urine dipstick for three consecutive days $(15,16)$.

All controls were healthy children who had non-infective, nonrenal conditions such as mild iron-deficiency anemia. All controls were assessed by a pediatrician and had no proteinuria, normal serum albumin level, and a serum creatinine within the normal ranges of $15-80 \mu \mathrm{mol} / \mathrm{l}$ (Table 1 ).

Table 1. Clinical and laboratory data of patients with INS and controls

\begin{tabular}{lccc}
\hline & $\begin{array}{c}\text { Patients with } \\
\text { INS }(n=15)\end{array}$ & $\begin{array}{c}\text { Controls } \\
(n=15)\end{array}$ & $\begin{array}{c}\text { Normal } \\
\text { range }\end{array}$ \\
\hline Median age (years) & $6.6 \pm 4.5$ & $6.8 \pm 3.5$ & \\
Gender & & & \\
$\quad$ Male (8) & & & \\
$\quad$ Female (7) & & & \\
Mean serum creatinine ( $\mu \mathrm{mol} / \mathrm{l})$ & $51 \pm 13$ & $49 \pm 11$ & $(15-88)$ \\
Mean serum protein $(\mathrm{g} / \mathrm{l})$ & $52 \pm 3^{\mathrm{a}}$ & $69 \pm 6$ & $(68-80)$ \\
Mean serum albumin $(\mathrm{g} / \mathrm{l})$ & $19 \pm 2^{\mathrm{a}}$ & $38 \pm 6$ & $(35-45)$ \\
Mean eGFR (ml/min/1.73 M²) & $160 \pm 28^{\mathrm{a}}$ & $108 \pm 10$ & $(90-120)$ \\
Mean serum cholesterol $(\mathrm{mmol} / \mathrm{l})$ & $6.1 \pm 1.1^{\mathrm{a}}$ & $3.0 \pm 0.52$ & $(3.1-5.2)$ \\
Mean u.PCR (mg/mmol) & $430 \pm 56^{\mathrm{a}}$ & $11.0 \pm 0.5$ & $(<20)$
\end{tabular}

INS, idiopathic nephrotic syndrome; u.PCR, urinary protein:creatinine ratio.

${ }^{a} P<0.05$ when compared with controls.
The inclusion criteria of patients with INS included newly diagnosed patients with INS or a steroid-sensitive patient with INS who develops relapse and has been off steroid treatment for more than 2 months before relapse to eliminate any steroid effect on the oxidative parameters under study. Steroid responsiveness was defined as an attainment of complete remission within the initial 4 weeks of full-dose corticosteroid therapy (15). Secondary and congenital nephrotic syndrome cases, if any, were excluded from the study. None of the patients with INS included in this study had any acute infection or a systemic disease.

Clinical data and blood samples were collected from patients and the 15 age- and sex-matched controls. Blood samples were drawn according to the protocols set by the ethical committee on use of human tissues. All patients and controls included in this study were not on any medication including prednisolone.

The study was conducted according to the Helsinki declaration criteria, and the approval has already been obtained from the Health Sciences Center's Committee for the protection of Human Subjects in Research and the Ministry of Health's Ethics Committee. An informed consent was obtained from patients or their parents and the controls as per guidelines of the Committees.

\section{Cell Culture and Biochemical Assays}

Peripheral blood mononuclear cells were isolated from patients and controls, and cultured in growth medium (RPMI 1640 containing $10 \%$ FBS, and penicillin and streptomycin, $5 \mathrm{U} / \mathrm{ml}$ and $5 \mu \mathrm{g} / \mathrm{ml}$, respectively) in a humidified atmosphere of $5 \% \mathrm{CO}_{2}-95 \%$ air according to a modified method of Haller and co-workers (17). Briefly, whole blood was diluted (1:1) with buffered saline and subjected to ficoll isopaque-gradient centrifugation, and lymphocytes were collected from the interphase and washed with RPMI 1640. After washing, the cells were resuspended in RPMI 1640 growth medium, placed into coated culture flasks, and cultured at $37^{\circ} \mathrm{C}$ in a cell culture incubator. Purity and yield of the isolated non-adherent lymphocytes were determined using cell-surface staining techniques. Cultured lymphocytes isolated from patients and healthy control subjects were centrifuged at $4{ }^{\circ} \mathrm{C}$, and cell pellet was homogenized in $0.25 \mathrm{~m}$ sucrose buffer, $\mathrm{pH}$ 7.2. Cell homogenates were then used for various biochemical assays.

\section{Enzyme Assays}

NADPH oxidase activity was measured in cell homogenates at $37^{\circ} \mathrm{C}$ using lucigenin and NADPH as described elsewhere (18). Briefly, cell homogenates were added to a reaction mixture containing $50 \mathrm{~mm}$ phosphate buffer, $\mathrm{pH}$ 7.1, $0.01 \mathrm{mM}$ EDTA, and $25 \mu \mathrm{M}$ lucigenin, and the reaction was started by adding $100 \mu \mathrm{M}$ NADPH. Chemiluminescence was recorded over a period of $3 \mathrm{~min}$, and specific activity was calculated as relative light units emitted per s/mg protein. Catalase activity was assayed using hydrogen peroxide as a substrate according to the method described earlier by Baudhuin et al. (19). Activity of total superoxide dismutase (SOD) in cell homogenates was assayed using a method (20) based on inhibition of nitro blue tetrazolium (NBT) dye reduction by SOD. One unit of SOD enzyme activity is defined as the amount of protein required for $50 \%$ inhibition of NBT reduction.

\section{Lipid Peroxidation Assay}

Plasma and total cellular levels of malondialdehyde (MDA), an index of lipid peroxidation, were measured using an assay kit from Calbiochem.

\section{Western Blot Analysis}

Cell homogenates were prepared and SDS-polyacrylamide gel electrophoresis (SDS-PAGE) was performed using Laemelli's sample buffer. Following SDS-PAGE, proteins were transferred on to nitrocellulose and immunoblotted with antibodies against NOX-1 and actin. Protein bands were visualized using horse raddish peroxidase (HRP)-labeled secondary antibody and ECL reagents. 


\section{NOX impairs PDGF activity Articles}

Bromo-Deoxyuridine (BrdU) Incorporation Assay

Bromo-deoxyuridine (BrdU) incorporation was measured as an index of DNA synthesis in cultures of lymphocytes established after treatment with experimental agents (FBS or PDGF in the presence or absence of DPI). Cells were growth-arrested for $48 \mathrm{~h}$ in a serum (mitogen)-free medium before addition of experimental agents. BrdU was added $2 \mathrm{~h}$ after the addition of growth factors to serumstarved cells, and incorporation of BrdU into lymphocytic DNA was measured $24 \mathrm{~h}$ after addition of mitogens using Calbiochem assay kit.

Amount of protein in cell homogenates was measured using Protein assay kit from Bio-Rad (Hercules, CA, USA).

\section{Statistical Analysis}

Data are presented as mean $\pm S D$, and the results were analyzed using Graph-pad Prism software (Prism software, San Diego, CA, USA). Significance of variance between two groups of the study was determined using student's $t$-test.

\section{RESULTS}

Clinical and biochemical data of 15 patients with INS recruited in this study and those of 15 controls are summarized in Table 1 . Out of the 15 patients with INS, two were newly diagnosed nephrotic patients with their first presentation at the time of study. The other 13 patients were steroid-sensitive frequent relapse patients who presented with relapse but were off steroid therapy for more than 2 months prior to the current relapse.

\section{NOX Protein and Enzyme Activity}

Figure 1a shows that PBL isolated from patients with NS had significantly $(P<0.01)$ higher activity of NOX as compared with control subjects. The observed increase in NOX activity was accompanied by a significantly higher $(P<0.01)$ protein expression of NOX-1 in cells isolated from patients with NS (Figure 1b).

\section{Catalase and SOD Activities}

Activities of antioxidant enzymes, catalase and SOD, are shown in Figures $2 \mathbf{a}$ and $\mathbf{b}$, respectively. Activity of catalase enzymes was found to be significantly $(P<0.01)$ less in $\mathrm{PBL}$ isolated from patients with NS in comparison with controls, whereas SOD enzyme activity in PBL of patients with NS was not significantly different from control cells.

\section{Malondialdehyde Levels}

As a measure of oxidative stress in PBL, Figure 3 shows levels of malondialdehyde (MDA), an index of lipid peroxidation. Cells isolated from patients with NS were observed to have significantly $(P<0.01)$ increased levels of MDA as compared with the control cells.

Mitogen-induced BrdU incorporation into PBL. Experiments carried out to examine the effect of growth factors on lymphocytes revealed that mitogen-induced DNA synthesis was significantly $(P<0.01)$ impaired in patients with INS (Figures 4 and 5). As shown in Figure 4, ex vivo BrdU incorporation in FBS-stimulated PBL of patients with INS was significantly lower as compared with the control counterparts. PDGF-induced BrdU incorporation was also significantly a
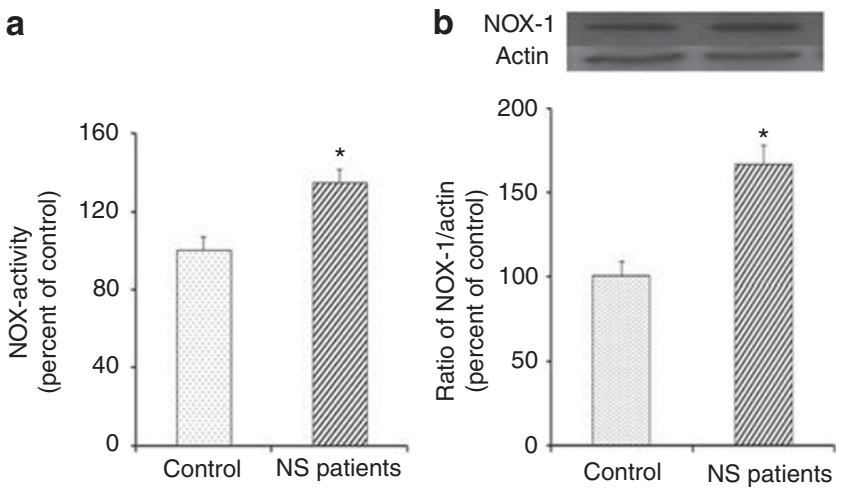

Figure 1. NADPH oxidase (NOX) activity (a) in peripheral blood lymphocytes (PBL) isolated from patients with nephrotic syndrome (NS) and controls. Results are presented as percent of control activity. Values are mean $\pm S D$ of at least six determinations. ${ }^{*} P<0.01$ when compared with controls. NOX-1 protein (b) content in PBL homogenates of patients with NS and controls is shown as a ratio with actin protein content. ${ }^{*} P<0.01$ when compared with controls.
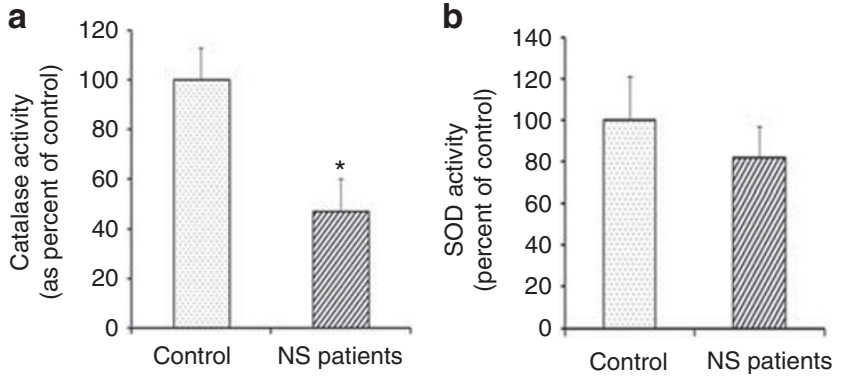

Figure 2. Enzyme activity of catalase (a) in peripheral blood lymphocytes (PBL) isolated from patients with nephrotic syndrome (NS) and controls. Results are presented as percent of control activity. Values are mean \pm SD of at least six determinations. ${ }^{*} P<0.01$ when compared with controls. Total superoxide dismutase (b) activity in PBL isolated from patients with NS and controls. Results are presented as percent of control activity. Values are mean $\pm S D$ of at least six determinations.

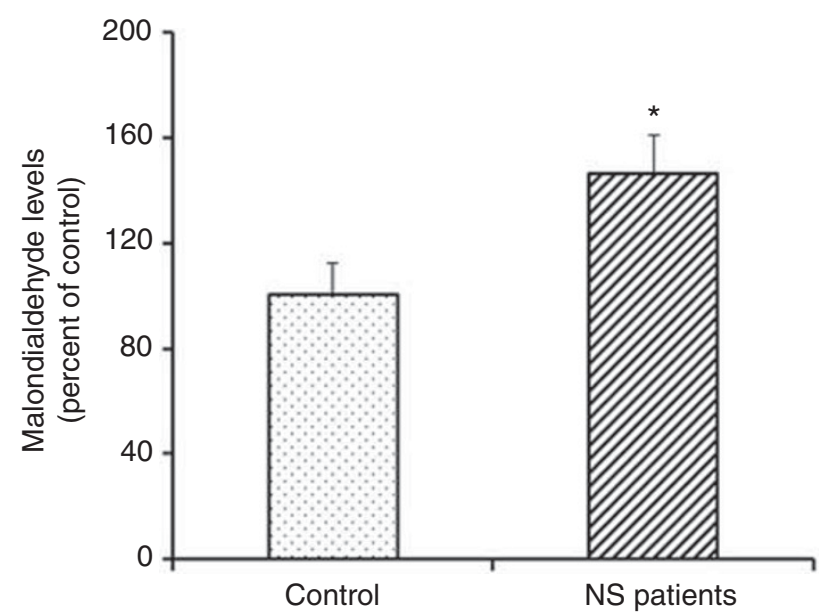

Figure 3. Levels of malondialdehyde (MDA) in cellular homogenates of peripheral blood lymphocytes (PBL) isolated from patients with nephrotic syndrome (NS) and controls. Results are presented as percent of control. Values are mean \pm SD of at least six determinations. ${ }^{*} P<0.01$ when compared with controls. 


\section{Articles | Al-Eisa and Dhaunsi}

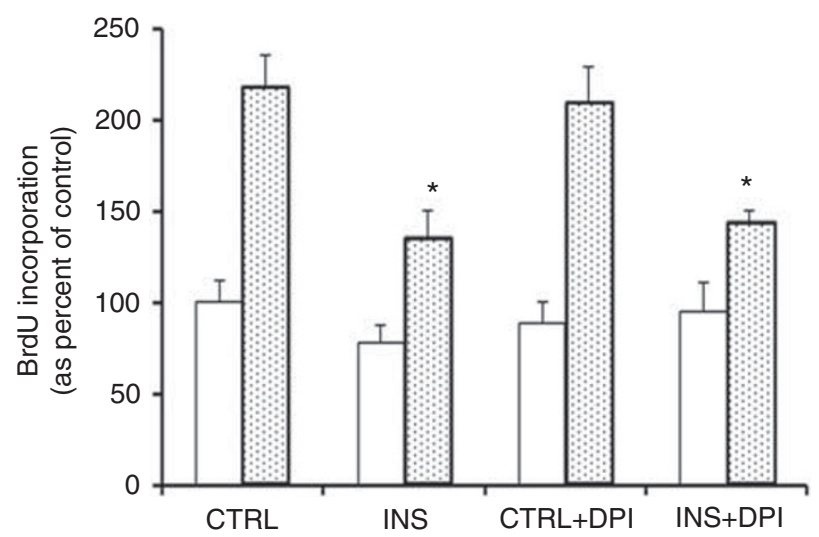

Figure 4. Bromo-deoxyuridine (BrdU) incorporation in lymphocytes from control subjects and patients with idiopathic nephrotic syndrome (INS) in a mitogen-free medium (MEM, open bars), and in response to $24 \mathrm{~h}$ treatment with $5 \%$ fetal bovine serum (FBS, shaded bars) in the presence or absence of NADPH oxidase (NOX) inhibitor, DPI. Values are mean \pm SD of six assays. ${ }^{*} P<0.01$, when INS or INS+DPI groups were compared with control FBS-stimulated lymphocytes.

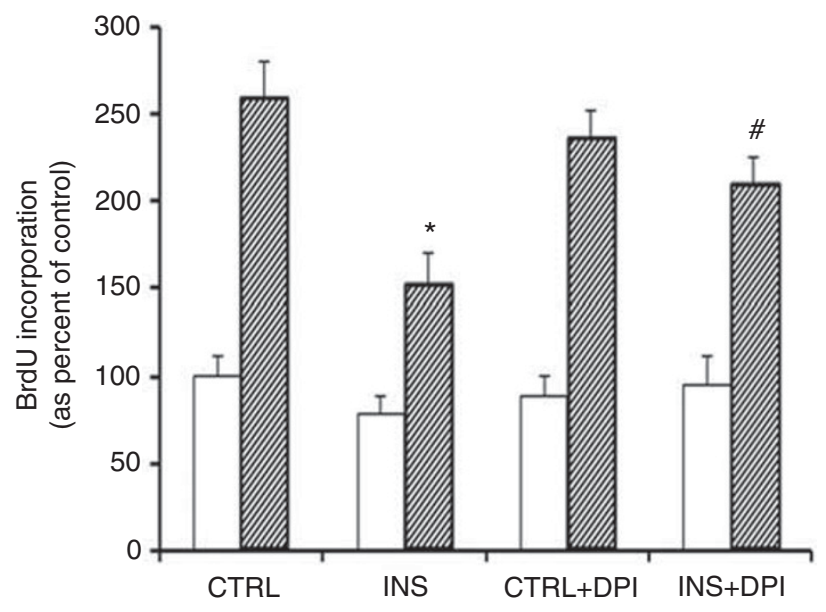

Figure 5. Bromo-deoxyuridine (BrdU) incorporation in lymphocytes from control subjects and patients with idiopathic nephrotic syndrome (INS) following $24 \mathrm{~h}$ incubation in a mitogen-free medium (MEM, open bars), and treatment with $50 \mathrm{ng} / \mathrm{ml}$ of platelet-derived growth factor (PDGF, shaded bars) in the presence or absence of NOX inhibitor, DPI. Values are mean \pm SD of six determinations. ${ }^{*} P<0.01$ when INS group was compared with control fetal bovine serum (FBS)-stimulated lymphocytes. ${ }^{\#} P<0.01$, when the INS+DPI group was compared with the INS group.

reduced in PBL of patients with INS (Figure 5); however, cotreatment of PBL with DPI, an inhibitor of NOX, markedly restored PDGF-induced DNA synthesis. DPI did not have any significant effect on FBS-induced DNA synthesis in PBL of patients with INS or controls.

\section{DISCUSSION}

INS is the most common renal disease among children. Although vast literature is available regarding incidence, clinical presentation, and treatment of NS, yet exact mechanism(s) of the onset and disease progression remain unknown. Besides underlying genetic factors and immunological events, development of cellular oxidative stress has been implicated in pathogenesis of NS. In this study, we demonstrate that NOX may be involved, at least partly, in regulating function of lymphocytes through ROS-mediated impairment of PDGF activity during onset and/or progression of NS.

NOX is now well known for its multitude role in pathophysiology of various conditions involving cardiovascular, renal, nervous system, respiratory, and immune systems through regulation of cellular oxidative stress (21). NOX, a major source of superoxide anion production, serves as a key player in maintaining levels of cellular ROS. Our findings that enzymatic activity of NOX is significantly more in PBL of patients with NS as compared with the control counterparts, would indicate blood lymphocytes being at a higher risk of cellular oxidative in children with NS. It has been reported earlier that renal NOX is involved in progression of the disease in patients with NS. Although renal NOX activity would be a true reflection of assessing the, in vivo, oxidative stress and its effects on kidneys during progression of the NS, yet measurement of the NOX activity in PBL, instead of an invasive and unavailable approach to obtain renal tissue from patients, provides important information about the role of lymphocyte-mediated responses and the overall status of oxidative stress in patients with NS. The fact that NOX-1 protein is significantly more in PBL of our patients with INS as compared with the control subjects suggests that either patients with NS may constitutively have higher cellular NOX or it may be induced in response to proinflammatory events in patients with NS. The fact that all patients recruited in this study were not taking any steroid treatment for a period of at least 2 months, there would be an enhanced risk of inflammation and production of certain proinflammatory cytokines, which are known to activate NOX (22). The efficacy of steroids and immunosuppressive treatments in INS implies the involvement of cytokines and immunogenic molecules in pathophysiology of the disease (23). An increase in the expression of Toll-like receptors and interleukin-6 has been linked to INS pathogenesis, and activation of NADPH oxidase has been independently reported to occur through Toll-like receptors and cytokines $(24,25)$. An increased activity of NOX through increased expression of NOX gene in patients with INS would indicate higher cellular oxidative stress in PBL that may impair the structure and function of blood lymphocytes to compromise the immune system. Impairment of antioxidant enzymes such as catalase, myeloperoxidase, and SOD in plasma/serum of patients with INS has also been reported earlier (26). Increased levels of malondialdehyde, an index of lipid peroxidation, observed in our patients support earlier studies that suggest an increased cellular oxidative stress in NS. Diminished catalase activity in PBL of our patients with INS accompanied by a moderate decrease in SOD activity would further indicate impairment of antioxidant defense system. A nonsignificant impairment of SOD activity in PBL of patients 
with INS could be the result of a possible induction of SOD to neutralize large amounts of superoxide generated by elevated NOX. A significant enhancement of NOX activity coupled with an impaired antioxidant enzyme system as observed in our patients with INS would indicate significantly elevated level of cellular oxidative stress in the peripheral blood cells. It has been reported earlier that lymphocytes undergo apoptotic changes in patients with INS and compromise the immune function of circulating blood cells. Findings of our study, possibly, provide an additional mechanism by which lymphocyte function is impaired through ROS-mediated downregulation of PDGF mitogenic activity. Activation of a major superoxide generating enzyme, NOX, in addition to impairment of antioxidant defense enzymes in PBL provides a challenging environment to the blood cells, which could trigger immunopathological events in kidneys of the patients with NS because of impaired growth and function of lymphocytes. Observed decrease in PDGF activity in patients with INS under conditions of elevated oxidative stress is intriguing, which could be either because of diminished levels of PDGF receptor or downstream signaling events, and nevertheless provides a new insight into possible mechanisms of INS pathogenesis.

Although the number of patients with INS in this study was small and the experimental studies were limited to the use of PBL, our findings provided valuable insights into molecular mechanisms of IgA-mediated nephropathy without invasive and sometimes unethical collection of renal tissues.

In conclusion, this study shows that NOX-mediated oxidative stress in blood lymphocytes and impairment of PDGF activity might have a role in immunopathology of INS and relapse in the absence of immunosuppressive/steroid therapy, thereby providing some new pathogenic mechanisms of INS.

\section{ACKNOWLEDGMENTS}

We thank Nini Mathew and Heba Dalvi for their technical support.

\section{STATEMENT OF FINANCIAL SUPPORT}

This study was supported by Kuwait University.

Disclosure: The authors declare no conflict of interest.

\section{REFERENCES}

1. Koomans HA. Pathophysiology of oedema in idiopathic nephrotic syndrome. Nephrol Dial Transplant 2003;18:30-2.

2. Gipson DS, Massengill SF, Yao L, et al. Management of childhood onset nephrotic syndrome. Pediatrics 2009;124:747-57.

3. Prasad N, Jaiswal AK, Agarwal Y, et al. Differential alteration in peripheral T-regulatory and T-effector cells with change in P-glycoprotein expression in childhood nephrotic syndrome: a longitudinal study. Cytokine 2015;72:190-6.

4. Kinra S, Rath B, Kabi BC. Indirect quantification of lipid peroxidation in steroid responsive nephrotic syndrome. Arch Dis Child 2000;82:76-8.
5. Mishra OP, Gupta AK, Prasad R, et al. Antioxidant status of children with idiopathic nephrotic syndrome. Pediatr Nephrol 2011;26:251-6.

6. Fan A, Jiang X, Mo Y, et al. Plasma levels of oxidative stress in children with steroid-sensitive nephrotic syndrome and their predictive value for relapse frequency. Pediatr Nephrol 2016;31:83-8.

7. Zachwieja J, Bobkowski W, Zaniew M, et al. Apoptosis and antioxidant defense in the nephrotic syndrome. Pediat Nephrol 2003;18:1116-21.

8. Zachwieja J, Dworacki G, Bobkowski W, et al. Increased apoptosis of peripheral blood lymphocytes in children with nephrotic syndrome. Pediatr Nephrol 2002;17:197-200.

9. Stepniewska J, Golembiewska, Dolegowska B, et al. Oxidative stress and antioxidative enzyme activities in chronic kidney disease and different types of renal replacement therapy. Curr Protein Pept Sci 2015;16:243-8.

10. Ueda $\mathrm{A}$, Hirayam $\mathrm{A}$, Nagase $\mathrm{S}$, et al. In vivo detection of intrinsic reactive oxygen species using acyl-protected hydroxylamine in puromycin nephrosis. Free Radic Res 2007;41:823-8.

11. Kamireddy R, Kavuri S, Devi S, et al. Oxidative stress in pediatric nephrotic syndrome. Clin Chim Acta 2002;325:147-50.

12. Quinn MT, Ammons MC, Deleo FR. The expanding role of NADPH oxidases in health and disease: no longer just agents for death and destruction. Clin Sci 2006;111:1-30.

13. DeLeo FR, Quinn MT. Assembly of the phagocyte NADPH oxidase, molecular interaction of oxidase proteins. J Leukoc Biol 1996;60:677-89.

14. Sorescu D, Szocs K, Griendling KK. NADP $(H)$ oxidases and their relevance to atherosclerosis. Trends Cardiovasc Med 2001;11:124-31.

15. Cattran DC, Feehally J, Terence Cook H, et al. Kidney Disease: Improving Global Outcome (KDIGO) Glomerulonephritis Work Group. KDIGO Clinical practice guidelines for glomerulonephritis. Kidney Int 2012;2: 139-274.

16. The primary nephrotic syndrome in children. Identification of patients with minimal change nephrotic syndrome from initial response to prednisone. A report of the International Study of Kidney Disease in Children. J Pediatr 1981;98:561-4.

17. Haller D, Blum S, Bode C, et al. Activation of human peripheral blood mononuclear cells by non-pathogenic bacteria in vitro: evidence of NK cells as primary targets. Infect Immun 2000;68:752-9.

18. Rajagopalan S, Kurz S, Münzel T, et al. Angiotensin II-mediated hypertension in the rat increases vascular superoxide production via membrane NADH/NADPH oxidase activation. Contribution to alterations of vasomotor tone. J Clin Invest 1996;97:1916-23.

19. Baudhuin P, Beaufay Y, Rahman LY, et al. Tissue fractionation studies. Intracellular distribution of monoamine oxidase, aspartate aminotransferase, alanine aminotransferase, D-amino acid oxidase and catalase in rat liver tissue. Biochem J 1964;92:179-84.

20. Spitz DR, Oberley LW. An assay for superoxide dismutase in mammalian tissue homogenates. Anal Biochem 1989;179:8-18.

21. Wen X, Wu J, Wang F, Liu B, Huang C, Wei Y. Deconvoluting the role of reactive oxygen species and autophagy in human diseases. Free Radic Biol Med 2013;65:402-10.

22. Hordijk PL. Regulation of NADPH oxidases: the role of Rac proteins. Cir Res 2006;98:453-62.

23. Xie $\mathrm{H}$, Fang $\mathrm{M}$, Lin $\mathrm{H}$, et al. Intermittent high-volume hemofiltration promotes remission in steroid resistant idiopathic nephrotic syndrome. Ren Fail 2015;37:966-73.

24. Pan Q, Wu J, Tao J, et al. Role of basophils in the pathogenesis of minimal change nephrotic syndrome: a literature review. Exp Ther Med 2014;8: 1027-31.

25. Lassegue B, Griendling KK. NADPH oxidases: functions and pathologies in the vasculature. Arterioscler Thromb Vasc Biol 2010;30:653-1.

26. Begenik H, Soyoral YU, Erkoc R, et al. Serum malondialdehyde levels, myeloperoxidase and catalase activities in patients with nephrotic syndrome. Redox Rep 2013;18:107-2. 